\title{
EVALUATION OF FOAM SCLEROTHERAPY WITH LIGATION OF SAPHENOFEMORAL JUNCTION VERSUS FOAM SCLEROTHERAPY ALONE IN TREATMENT OF VENOUS LEG ULCERS
}

\author{
By \\ Abd El-Aziz A. Abd El-Hafez, Hany Abd El-Momein, Mohamed \\ Shalamesh*, Mohammed M. Asar* and Mohammad Abol-Wafa** \\ Departments of Vascular Surgery, *General Surgery and **Radiology, Faculty of \\ Medicine, Al-Azhar University
}

Corresponding author: Abd El-Aziz Ahmed Abd El-Hafez,

Mobile: (+20)01000004564, E-mail: aziz4002@hotmail.com

\begin{abstract}
Background: Venous leg ulcers (VLUs) are common chronic recurrent, conditions, and major causes of morbidity and disability. VLUs usually associated with chronic venous insufficiency. Foam sclerotherapy (FS) has been applied to the treatment of a variety of venous conditions because of its efficacy, efficiency, low cost and minimally invasive nature of the procedure. Surgical correction of superficial, perforating, or deep-venous disease may be needed for healing of venous ulcer.
\end{abstract}

Objective: To evaluate the clinical efficacy of saphenofemoral junction ligation with FS in comparison with FS alone in treatment of patients associated with chronic venous insufficiency that had isolated superficial venous and perforators reflux and complicated by VLUs.

Patients and Methods: The present study included 40 patients with VLUs associated by isolated superficial venous and perforators reflux. They presented to the Vascular Surgery Department of Al-Azhar University Hospitals, Cairo, Egypt, over a 5- years period from November 2015 to October 2020. Patients were divided into two equal groups: group A underwent FS ulcer related perforating veins only, and group B underwent ligation of saphenofemoral junction and FS. The two groups were followed by compression. The patients were followed up for ulcer healing time, rate of healing, and the rate of recurrence for at least 12 months.

Results: The patient population was thirty-two males (80\%) and eight females (20\%), with VLUs and superficial venous system insufficiency over a 5-years period. In group A, thirteen patients $(65 \%)$ reached the end point of adequate healing or complete healing within 14-24 weeks, while in group B sixteen patients (80\%) reached the end point within 8-16 weeks. The recurrence rates at 12 months were $30 \%$ (six patients) in group A and 10\% (two patients) after surgery in group B.

Conclusion: Ligation of the saphenofemoral junction with FS can improve the outcomes of patients with venous ulcers in comparison with FS alone.

Keywords: Venous leg ulcers, Foam sclerotherapy, saphenofemoral junction ligation and chronic venous insufficiency.

\section{INTRODUCTION}

Chronic venous insufficiency (CVI) can be defined as an abnormally functioning venous system of the lower extremities caused by venous valvular incompetence with or without associated venous outflow obstruction. Characterized by the retrograde flow of blood in the 
lower extremity, resulting in venous hypertension in the distal venous system of the lower extremity, causing various pathologies, including pain, edema, skin changes, and ulcerations. Venous leg ulcers (VLUs) are strongly associated with roughly, two main causes. Deep venous insufficiency (common in postthrombotic syndrome; less common in primary deep venous insufficiency) and superficial venous Insufficiency), usually varicose veins (O'Brien et al., 2018).

VLUs represent a major burden to healthcare services affecting at least $1 \%$ of the population. Because of the substantial morbidity and the financial and psychosocial costs involved, it is important to identify the most effective means of treatment for venous ulceration. The progressive development and modification of treatments are essential to overcome the natural history of prolonged ulceration and recurrence that make venous ulceration extremely difficult to cure (Glovicki et al., 2014).

The Identification of the site of venous disease in patients with venous ulcer with duplex is important to decide the treatment modality. Duplex will define the origin of venous reflux causing the varicose veins and confirm the function and patency of the deep venous system. Duplex is superior to both clinical examination and hand-held Doppler for accurate assessment of venous reflux (Marsden et al., 2013).

Chronic venous disease is commonly stratified using the CEAP (Clinical, Etiology, Anatomy, Pathophysiology) classification, which grades venous disease on the basis of the presence of dilated veins, edema, skin changes, or ulceration. Chronic venous insufficiency is defined as CEAP 3 to 6 and represents advanced venous disease (Comerota et al., 2011).

There are different theories about the development of venouse ulcers .Most of them implies that the pressure in the capillaries is increased. Thus ambulatory capillary hypertension could be established as the primary pathophysipological condition forming the basis for most of the current theories about the ulcer formation. The occurrence of VLUs is strongly associated with venous disease (e.g. varicose veins and deep vein thrombosis), contributing to sustained venous hypertension (Mercer et al., 2016).

Conservative treatment in the form of limb elevation, compression therapy, wound care and pharmacological therapy is effective line of treatment of VLUs but with risk of recurrence. Surgical correction of superficial, perforating, or deep-venous disease may be needed for ulcer healing. The goal of surgical intervention is to improve venous hemodynamics when conservative therapy has failed (Simon, 2014).

Foam sclerotherapy has the advantage of real-time visualization as an effective contrast agent on ultrasound imaging, allowing manual displacement of the foam into the selected veins. Additionally, the minimally invasive nature of the procedure permits the patient to return immediately to daily activities. Because of its efficacy, efficiency, and low cost, foam sclerotherapy has been applied to the treatment of a variety of venous conditions. Among these is the treatment of venous ulcers (Evans et al., 2018). 
Foam sclerotherapy has no anatomical limitation, including ankle ulcers, and can be performed in open ulcers. Moreover, by a "sponge" effect, the injected foam is uniformly distributed to the adjacent area and besides closing the vessels with reflux, can also close all small pathways that transmit venous hypertension to the skin (Rosen et al., 2019).

Compression is an important part of the treatment to optimize the effect of foam sclerotherapy. Patients are advised to keep as active as possible, and to avoid dependence of the lower limbs and long periods of immobility (Dinn and Henry, 2012).

Previous studies have proved that more than $50 \%$ of patients have isolated superficial system reflux. In the ESCHAR study (comparison of surgery and compression with compression alone in venous ulceration: randomized controlled trial), $60 \%$ of the patients had isolated superficial reflux, in the Shami study 53\% of the patients had isolated superficial system reflux and in the study of Robertson et al. in 2014 confirm these effect of foam sclerotherapy in the treatment of venous ulcer, which accelerates ulcer closure, reduces the incidence of recurrences with minimal complications and cheapest cost (Robertson et al., 2014).

The purpose of this study was to evaluate the clinical efficacy of saphenofemoral junction ligation after FS in comparison with FS alone in treatment of patients with varicose veins and venous ulcers in lower extremities.

\section{PATIENTS AND METHODS}

This study included 40 patients with VLUs classified according to CEAP (Clinical, Etiology, Anatomy, and Pathophysiology). All the patients had CEAP 6 ulcers (indicating skin changes with active ulceration) associated by isolated superficial venous and perforators reflux long saphenous vein incompetence (reflex of saphenofemoral junction and ulcer related perforators) .They presented to the Vascular Surgery Department of AlAzhar University Hospitals Cairo, Egypt, over a 5- years period from November 2015 to October 2020 They were randomly distributed into two equal groups; a group A was treated with compression only and composed of 20 patients, while group B treated with surgery on the saphenous system in form of ligation of Saphenofemoral junction and foam sclerotherapy of perforating veins followed by compression and composed of 20 patients.

An approval of the study was obtained from Al- Azhar University academic and ethical committee. Every patient signed an informed written consent for acceptance to share in this research.

Inclusion criteria: Patients with only VLUs associated by isolated superficial venous and perforators reflux (reflex of saphenofemoral junction and ulcer related perforators) were included.

Exclusion criteria: Patients with other types of ulceration as malignant ulcers and autoimmune ulcers, ischemic limbs (i.e. rest pain), patients associated with deep venous affection (incompetence or obstruction), ankle brachial index (ABI) less than 8.5 , previous vasculitis, 
collagen-vascular diseases, and dermal manifestations of systemic diseases.

At the initial clinical presentation, full history was taken from every patient and the clinical data were prospectively collected regarding age, gender, risk factors and comorbidities. All the patients were assessed by color duplex ultrasound scanning to determine the location of the venous disease. ABI and arterial duplex were done. VLUs of all patients of the two groups were treated by daily basic wound care principles were followed, such as proper wound environment, control of clinical signs of infection, and debridement. Removal of all necrotic tissue, densely adherent slough, and exudates was carried out for all patients.

VLUs were followed up monthly. Post management, wound dressing protocol was standardized. Patients were followed up until they reached one of the end points of the study, which were a healed wound, a healing wound, non-healing wound and major amputation. A wound was considered completely healed if it was fully covered with intact skin. It was considered adequately healing if it was completely covered with healthy granulation tissue, with absence of tissue necrosis or infection. It was considered non-healed if it did not show signs of healthy granulation tissue during followup. During follow-up, data were collected regarding wound status, the details of the management plan and details of venous duplex scanning

Group A was treated with Foam sclerotherapy and compression only: Elastic, multilayered compression bandages were used. Compression bandage systems were wrapped around the leg from the foot to the upper calf, which provided a pressure of $20-40$ $\mathrm{mmHg}$. Care was taken to avoid excessive compression of skin against any bony prominences. The patients were instructed to walk to achieve the full compressive effect of the bandage. Foam sclerotherapy with compression was the mode of treatment in group $\mathrm{A}$ and used also in group B.

Group B treated with Ligation of saphenofemoral junction with foam sclerotherapy of perforating veins followed by compression: With general or Spinal anasthesia, a groin or a thigh incision to identify the saphenous vein. Saphenofemoral junction disconnection were performed with ligation of the tributaries. Foam sclerotherapy of perforating veins was done followed by compression.

\section{Statistical analysis:}

Data were collected, revised, coded and analyzed using statistical package for the social sciences (IBM SPSS) version 23.The quantitative data were presented as number (No.), percentage (\%), mean (X), standard deviation (SD) and range. The comparison between two independent groups with quantitative data and parametric distribution was done by using One Way Analysis of Variance (ANOVA). Significance level $(\mathrm{P})$ value: $\mathrm{P}$ value $<0.05$ was considered significant. 


\section{RESULTS}

The study included 40 limbs, belonging to 40 patients. The patient population consisted of 32 males $(80 \%)$ and eight females (20\%), with Mean age \pm SD $(32.6 \pm 7.2)$ years with VLUs and superficial venous system insufficiency over a 5-year period. Patients were divided into two equal groups: group $\mathrm{A}$ underwent compression only, and group B treated with surgery on the saphenous system in form of ligation of Saphenofemoral junction and foam sclerotherapy of perforating veins followed by compression. The patients were followed up for time of ulcer healing, rate of healing, and the rate of recurrence for at least 12 months.

The demographic characteristics and distribution of patient according to basic CEAP classification and ulcer distribution are shown in the following tables. The patient population consists of 32 males and 8 females with a mean age $32.6( \pm 7.2)$ (Table1).

Table (1): Baseline clinical characteristics of patients

\begin{tabular}{|c|c|c|c|}
\hline \multicolumn{2}{|c|}{ Variables } & No. & Percent (\%) \\
\hline \multirow{2}{*}{ Gender } & Male & 32 & 80 \\
\cline { 2 - 4 } & Female & 8 & 20 \\
\hline \multirow{3}{*}{ Age (year) } & Mean \pm SD & \multicolumn{2}{|c|}{$32.6 \pm 7.2$} \\
\cline { 2 - 4 } & Min. & \multicolumn{2}{|c|}{26} \\
\cline { 2 - 4 } & Max. & \multicolumn{2}{|c|}{42} \\
\hline
\end{tabular}

Forty lower limbs were included in this study of 40 patients (27 limbs were Lt sided while 13 were Rt sided) (Table 2).

Table (2): Distribution of venous ulcer in the lower limbs

\begin{tabular}{|c|c|c|}
\hline The affected limb & No. of patients & $\%$ \\
\hline Left lower limb & 27 & $67.5 \%$ \\
\hline Right lower limb & 13 & $32.5 \%$ \\
\hline Total & 40 & $100 \%$ \\
\hline
\end{tabular}

Patient's distributions according to 34 C6EpAs\&pPr, 3 C6EsAs\&pPo, 3 basic CEAP classification were 40 C6EsAs\&pPr,o according to pathological C6EsAs\&p\&d according to Clinico- classification (Table 3).

Etiological/ Anatomical classification and

Table (3): Patient's distribution according to basic CEAP classification

\begin{tabular}{|c|c|c|c|}
\hline C & E & A & P \\
\hline \multirow{2}{*}{ 40 C6 } & \multirow{4}{*}{ 40 C6Es } & \multirow{4}{*}{ 40 C6EsAs\&p\&d } & 34 C6EpAs\&pr \\
\cline { 4 - 4 } \cline { 3 - 4 } & & & 3 C6EsAs\&pPo \\
\cline { 4 - 5 } & & &
\end{tabular}

C1-6: Skin changes in conjunction with active ulceration, Ec: Congenital venous disease (VD), Ep: Primary VD, Es: secondary () VD,

As: superficial veins, Ap: perforator veins, Ad: deep veins

Pr: Reflux, Po: Obstruction, Pr.o: reflux and obstruction 
The procedure time was estimated from the time of infiltration of anaesthesia to the end of the procedure. It ranged from
20 min to 45 minutes in group A and from 45 min to 100 minutes in group B (Figure 1 and 2).

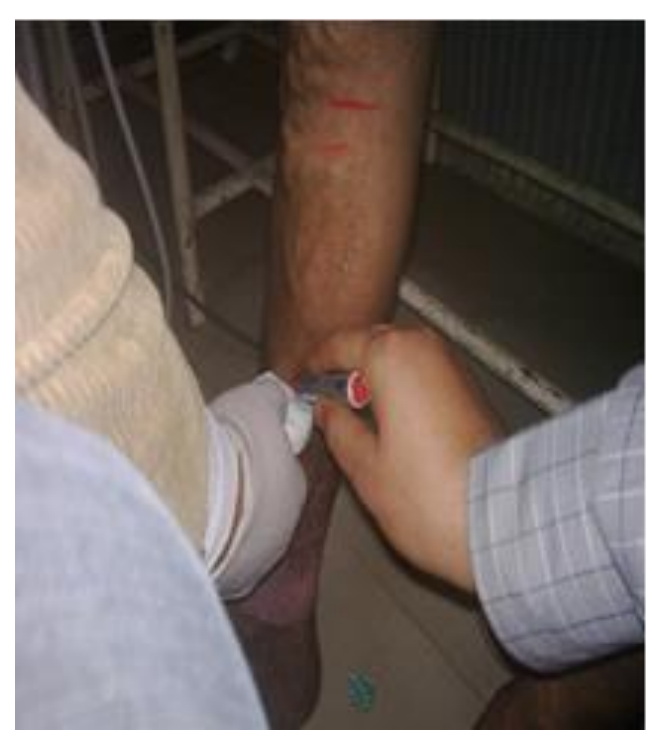

Figure (1): A case of duplex guided FS of perforator vein

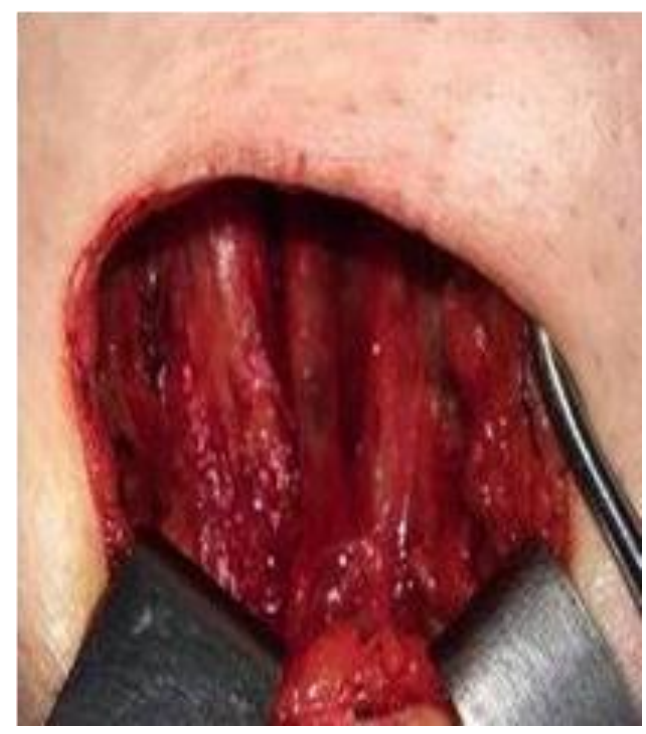

Figure (2): A case of exposure of GSV for ligation of Saphenofemoral junction 
$65 \%$ in group A (13 patients) within 14-24 weeks reached the end point of adequate healing or complete healing and $80 \%$ in group B (16 patients) within $8-16$ weeks. The recurrence rates at 12 months were $30 \%$ (six patients) in group A and $10 \%$ (two patients) after surgery in group $\mathrm{B}$ (Figure 3).

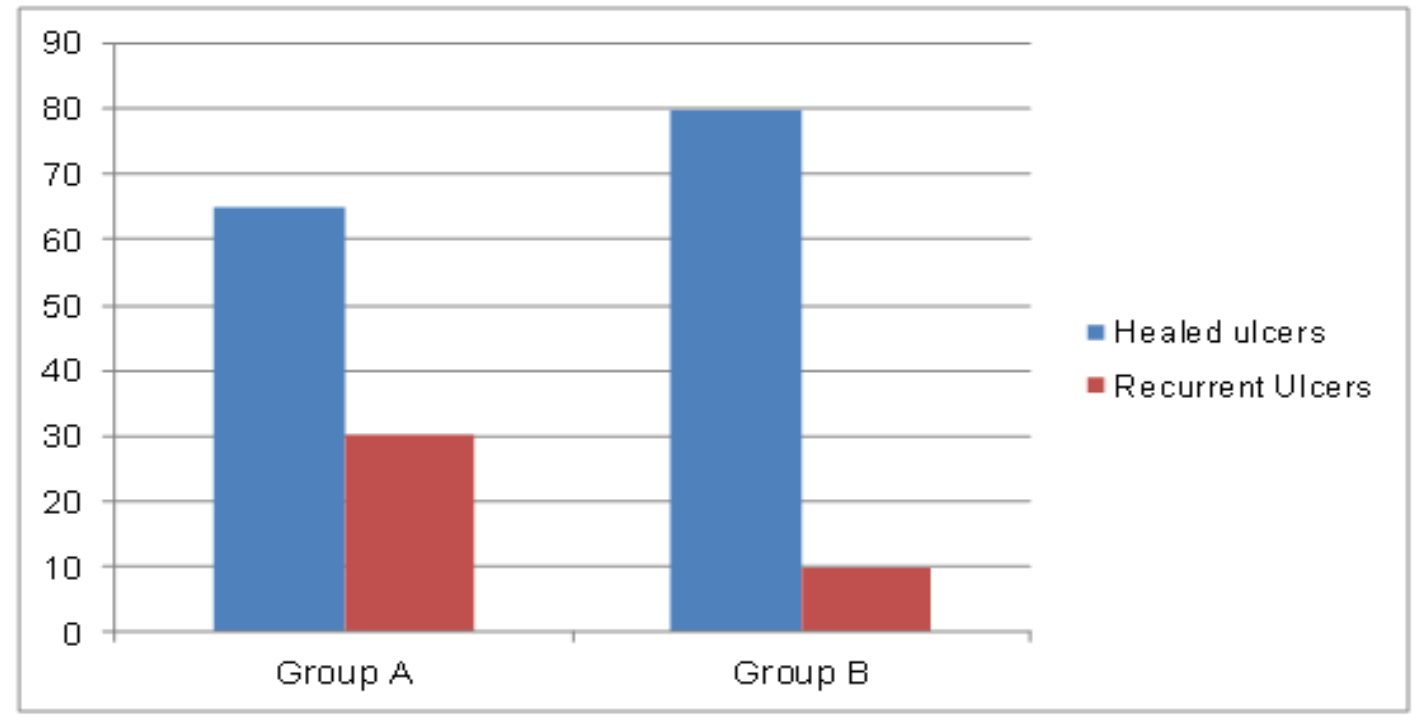

Figure (3): Healed and recurrent ulcers in group A and group B

\section{DISCUSSION}

The complicated natural history of venous ulcers requires continued development and improvement of treatments to ensure the most effective management.

VLUs represent the most severe manifestation of chronic venous insufficiency. VLU is a problem affecting thousands of patients annually (Simon, 2014). These ulcerations are known to be difficult to heal leading to negative effect on the patient's quality of life, with a significant economic burden on the healthcare system (Rosen NA, et al, 2014). Conservative treatment in the form of limb elevation, compression therapy, wound care and pharmacological therapy is effective line of treatment of VLU but with risk of recurrence (Dinn and Henry, 2012). Surgical correction of superficial, perforating, or deep-venous disease may be needed for ulcer healing. The goal of surgical intervention is to improve venous hemodynamics when conservative therapy has failed.

The occurrence of VLUs is strongly associated with venous disease, e.g. varicose veins and deep vein thrombosis), contributing to sustained venous hypertension (Nelson et al., 2012).

In over half of the patients with venous ulceration, the disease was confined to the superficial venous system. In their study, Shami (2014) found that isolated deep venous reflux was present in only $15 \%$, a combination of deep and superficial venous reflux was found in $32 \%$, and in $53 \%$ of their patients there was only superficial venous reflux. In the ESCHAR study, $60 \%$ of the patients had isolated superficial reflux, and in the Robertson 
study in 2014 two-third of the patients had superficial system reflux.

In our study, we selected patients with isolated superficial venous reflux to exclude the effect of deep venous incompetence on the outcome.

Over the years, clinicians have been faced with numerous treatment options for VLUs. The aim of this study was to evaluate the efficacy of superficial saphenous venous system surgery as a management for VLUs with superficial venous reflux.

Chronic venous disease is commonly stratified using the CEAP classification, which grades venous disease on the basis of the presence of dilated veins, edema, skin changes, or ulceration. Chronic venous insufficiency is defined as CEAP 3 to 6 and represents advanced venous disease (Comerota et al., 2011).

Being the cornerstone of management for patients with chronic venous insufficiency (venous valvular reflux), the use of elastic multilayered compression bandages was the mode of treatment in group A and used for postoperative care in group B in this study.

The results of this study showed that the rate of ulcer healing was higher and faster in group B (80\%) within 8-16 weeks compared with group A $(65 \%)$ within 14-24 weeks.

Results of ESCHAR study revealed no statistically significant difference in healing rates in patients managed with FS plus surgery and those managed with FS alone .In contrast, Taradaj et al. (2018) reported a statistically significant difference favoring surgery plus FS. The accepted statistics indicated that VLUs require an average of 24 weeks to heal (Goss et al., 2014).

Gohel and colleagues in 2019 concluded that surgical correction of superficial venous reflux does not increase healing rates in patients with VLUs receiving FS therapy. In 500 patients with open or recently healed VLUs and superficial venous reflux, healing rates at 3 years were $89 \%$ for the FS group and 93\% for the FS plus surgery group. This conclusion could be explained by the longer duration for FS (3 years) used.

However, in our study, the healing rate improved to $80 \%$ by surgery and the time of healing was reduced (8-16 weeks); the higher surgical success may be due to patient selection with isolated superficial saphenous incompetence. This is in agreement with the study of Goss et al. (2014) who reported a $100 \%$ healing rate for limbs without DVT or post-thrombotic syndrome, and with the study of Taradaj et al. (2018) who achieved $100 \%$ healing in patients with superficial venous incompetence alone.

When considering recurrence rates, in this study, the rates of ulcer recurrence at 12 months were lower in group B (10\%) (FS plus surgery) than in group A $(30 \%)$ (FS), and this was similar to both ESCHAR study and the Taradaj et al. (2018) who found lower incidence of recurrence among patients who underwent surgery in addition to FS as compared with patients who were managed with FS alone. In the ESCHAR study, there was a $12 \%$ recurrence rate at 1 year for patients managed with FS plus surgery, as compared with $28 \%$ in the group managed with FS alone. Recurrence rates at 4 years continued to favor surgery plus FS versus 
FS alone (31 vs. 56\%). Taradaj et al. (2018) also reported lower recurrence rates at 2 years after intervention in patients who underwent surgery, FS, and drug therapy compared with the group that received compression stockings and drug therapy. In contrast, Young et al. (2016) did not find any statistical difference in recurrence rates among patients treated with FS alone and those treated with FS plus surgery (23 and 22\%, respectively).These findings support the role of surgery in addition to FS therapy in patients with VLUs.

\section{CONCLUSION}

Ligation of the treated varicose veins after FS can improve the outcomes of patients with venous ulcers in comparison with FS alone. FS is a safe, effective, and technically feasible procedure and can be used as a day surgery.

\section{REFERENCES}

1. Comerota AJ, Valencia, Grace PA and Scott DJ. (2011): Chronic venous insufficiency of the lower extremity: definition, epidemiology, and economic and social burdens. Semin Vasc Surg., 18:6-7.

2. Dinn S and Henry BM. (2012): Diagnostic values of duplex imaging are required before all operations for primary varicose veins. Br J Surg., 85:1195-1197.

3. Evans CJ, Fowkes FGR, Ruckley CV and Lee AJ. (2018): Prevalence of varicose veins and chronic venous insufficiency in men and women in the general population: Edinburgh vein study, J Epidemiol Community Health. 53: 149-153.
4. Glovicki P, Comerota AJ, Dalsing MC, Eklof BG, Gillespie DL, Gloviczki ML and Sotet N. (2014): The care of patients with varicose veins and associated chronic venous disease: clinical practice guidelines of the Society for Vascular Surgery and the American Venous Forum. J Vasc Surg., 53(Suppl):2S-48S.

5. Gohel SR, Grace PA, Scott DJ and Grace PA. (2019): Chronic venous insufficiency of the lower extremity: definition, epidemiology, and economic and social burdens. Semin Vasc Surg., 18:6-7.

6. Goss DE, de Trafford J, Roberts VC and Fowkes FGR. (2014): Raised ankle/brachial pressure index in venous ulcer-treated patients. Diabet Med., 6:576-8.

7. Marsden G, Perry M, Kelley $K$ and Davies AH. (2013): Guideline Development Group. Diagnosis and management of varicose veins in the legs: summary of NICE guidance. BMJ, 347:4271-4279.

8. Mercer KG, Scott DJ and Berridge DC. (2016): Preoperative duplex imaging is required before all operations for primary varicose veins. Br J Surg., 85:1495-1497.

9. Nelson E, Cullum $\mathbf{N}$ and Jones $\mathbf{J}$. (2012): Venous leg ulcers. Clinical evidence, 7: 1806-1817.

10. O'Brien JF, Grace PA, Perry IJ and Burke PE. (2018): Prevalence and aetiology of leg ulcers in Ireland. Ir $\mathbf{J}$ Med Sci., 169:110-112.

11. Robertson S, Simon DA, Dix FP and McCollum CN. (2014): Management 
of venous leg ulcers. BMJ, 328:13581362.

12. Rosen NA, Iafrati $M D, M a H$ and O'Donnell TF. (2019): The real cost of treating venous ulcers in a contemporary vascular practice. J Vasc Surg Venous Lymphat Disord., 2014; $2: 355-361$.

13. Shami D. (2014): Venous ulcers of the lower extremity: epidemiology, physiopathology, diagnosis and treatment. Int J Dermatol. , 44:449456.

14. Simon BK. (2014): Venous ulcers of the lower extremity: definition, epidemiology, and economic and social burdens. Semin Vasc Surg., 28:3-5.
15. Taradaj J, Franek A, Cierpka L, Brzezinska-Wcislo L, Blaszczak E and Polak A. (2018): Early and longterm results of physical methods in the treatment of venous leg ulcers: randomized controlled trial. Phlebology; 26:237-245.

16. Young MJ, Adams JE and Anderson GF (2017): Management of venous leg ulcers: a prospective, randomized, multicenter trial. $\mathrm{J}$ Vasc Surg., 36:615-21. 
تقييم الحقن الرغوى مع الربط مقارنة بالحقن الرغوى بمفرده في علاج قر ح الساق الوريدية

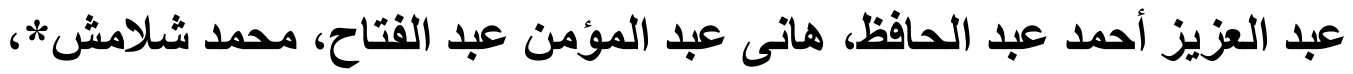

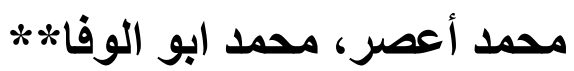

أقسام جراحة الأوعبة الاموية والجراحة العامة* والأثعة**، كلية الطب، جامعة الازهر

E-mail: aziz4002@hotmail.com

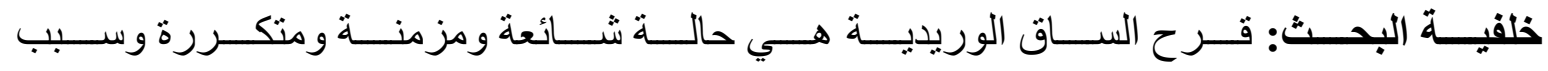

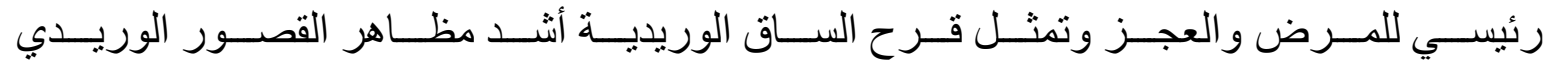

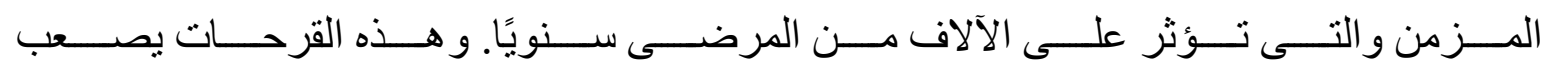

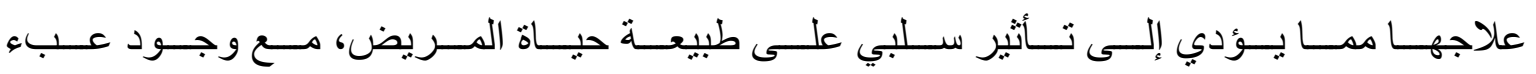

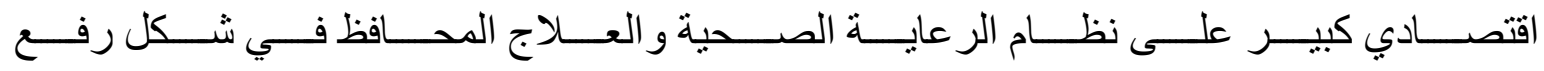

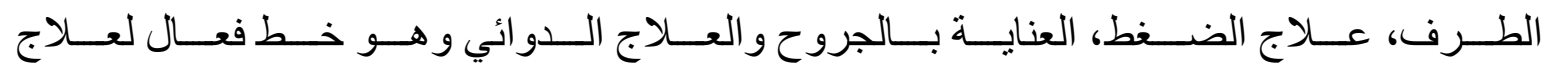

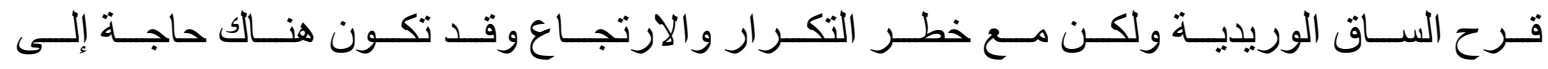

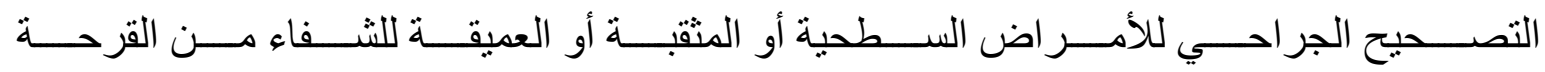

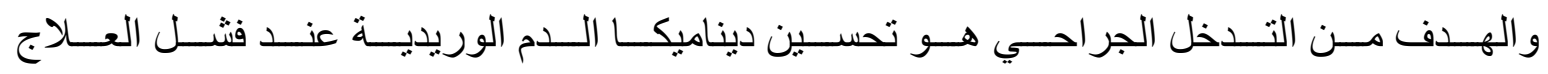

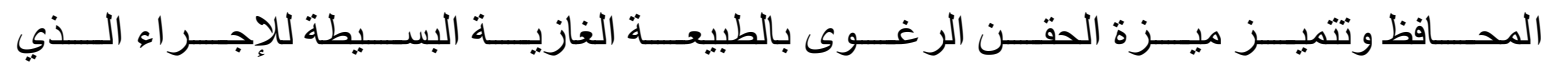

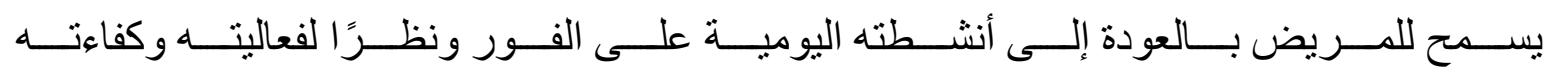

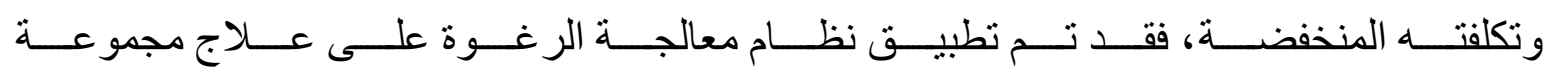
متنوعة من الحالات الوريدية ومن بين هذه علاج القرحة الوريدية .

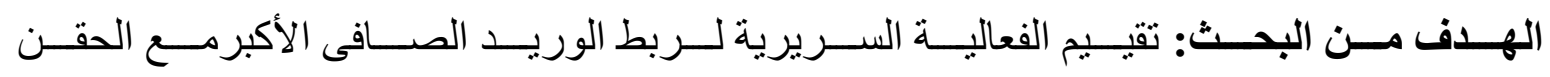

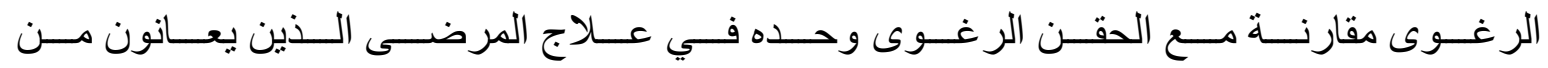
الدو الي و القرحة الوريدية في الأطر اف السفلية.

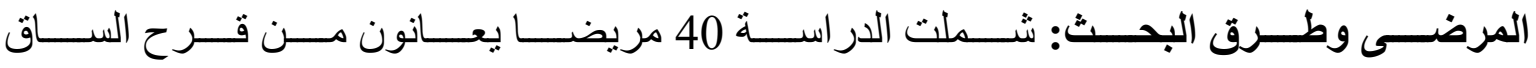

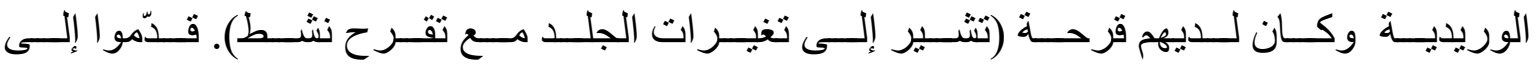

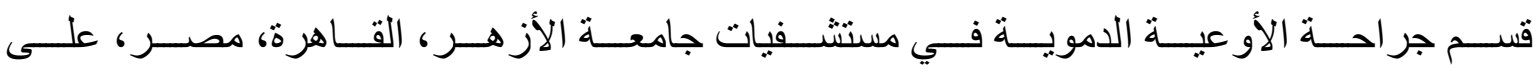

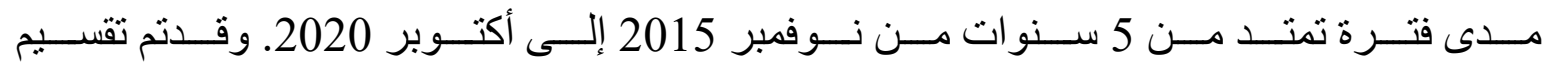




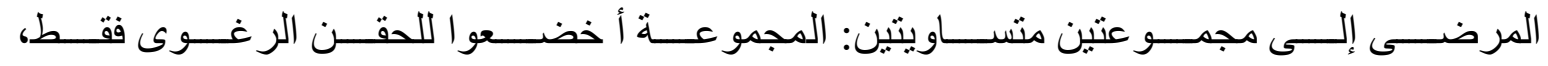

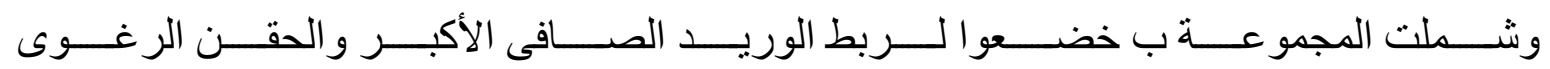

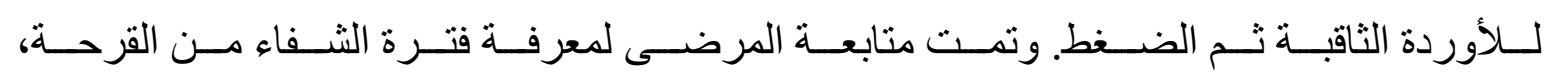
ومعدل الثفاء، ومعدل التكر ار لمدة 12 شهر الفلى وته الأقل.

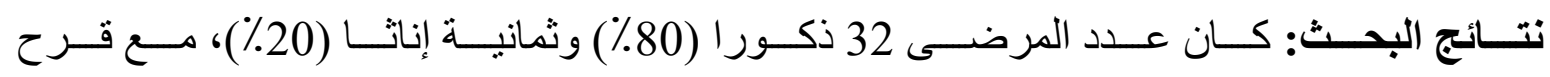

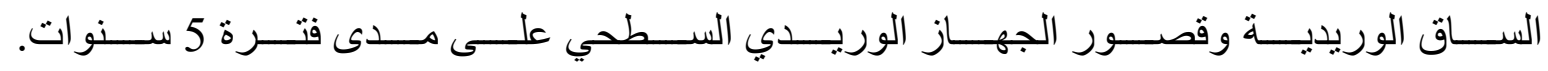

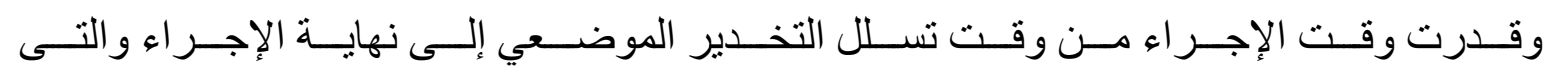

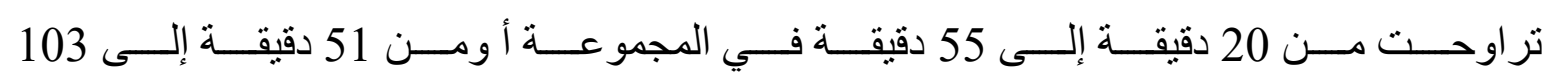

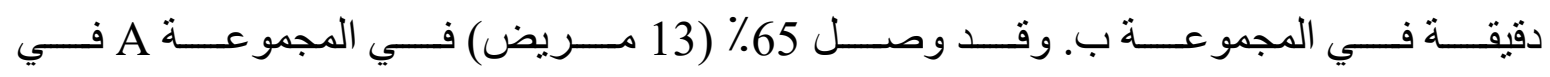

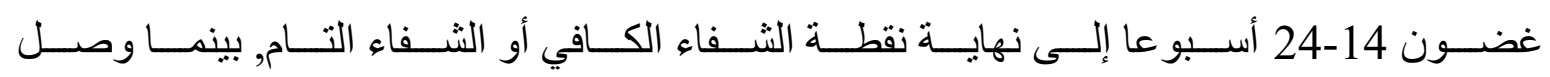

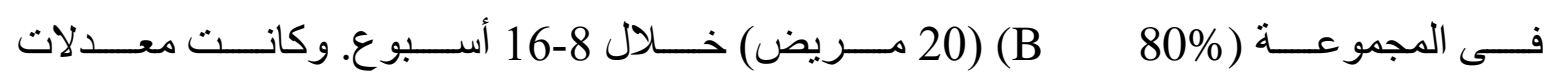

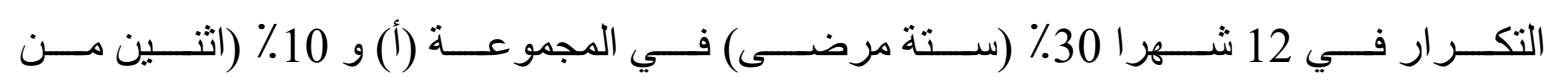

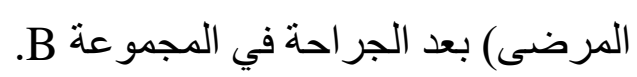

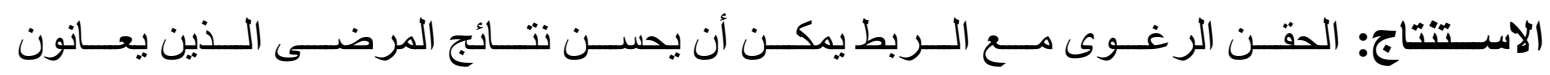

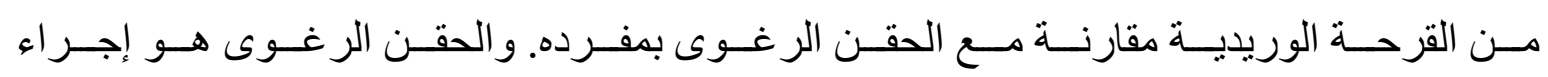
آمن وفعال وممكن من الناحية الفنية.

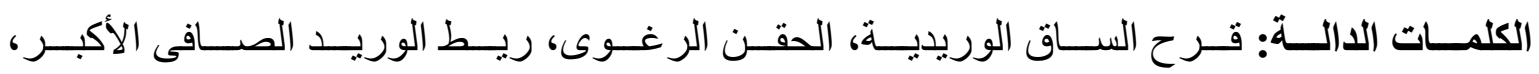

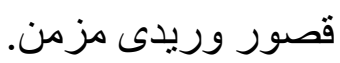

